\title{
Comparative study of human colonic tumor-derived endothelial cells (HCTEC) and normal colonic microvascular endothelial cells (HCMEC): Hypoxia-induced sVEGFR-1 and sVEGFR-2 levels
}

\author{
CAREN JAYASINGHE ${ }^{1,2 *}$, NEKTARIA SIMIANTONAKI ${ }^{1,2 *}$, \\ ROMI MICHEL-SCHMIDT ${ }^{1}$ and CHARLES JAMES KIRKPATRICK ${ }^{1}$ \\ ${ }^{1}$ Institute of Pathology, Johannes Gutenberg University, Langenbeckstrasse 1, 55131 Mainz, Germany
}

Received October 24, 2008; Accepted November 26, 2008

DOI: $10.3892 /$ or_00000306

\begin{abstract}
Colorectal carcinoma growth and progression is dependent on the vasculature of the tumor microenvironment. Tumor-derived endothelial cells differ functionally from their normal counterpart. For this reason we isolated microvascular endothelial cells from human colon cancer tissue (HCTEC) and compared them with endothelial cells from normal colonic tissue (HCMEC) of the same donor. Since hypoxia is a universal hallmark of carcinomas, we examined its effects on HCTEC of five patients in comparison with the corresponding HCMEC, with respect to the secretion of the soluble form of the two important vascular endothelial growth factor (VEGF) receptors, VEGFR-1 and -2. After dissociation by dispase/ collagenase of central non-necrotic tumor areas obtained from colon carcinomas, HCTEC were isolated using CD31coated magnetic beads and cultivated as monolayers. Subsequent characterization studies demonstrated the endothelial phenotype, including VEGFR-1 and -2 mRNA and protein expression as well as E-selectin expression, upregulated after LPS, TNF $\alpha$ and IL-1ß stimulation. sVEGFR expression analyses were performed using ELISA. In comparison with HCMEC markedly lower sVEGFR-1 protein concentrations were found in HCTEC. These low sVEGFR-1 levels remain unchanged under hypoxia. In contrast, sVEGFR-2 was significantly decreased in both HCMEC and HCTEC under hypoxic conditions ( $\mathrm{p} \leq 0.001)$. Comparative studies with endothelial cells isolated from
\end{abstract}

Correspondence to: Dr C. Jayasinghe or Dr N. Simiantonaki, Institute of Pathology Klinikum Leverkusen, Am Gesundheitspark 11, 51375 Leverkusen, Germany

E-mail: jayasinghe@klinikum-lev.de; simiantonaki@yahoo.de

Present address: ${ }^{2}$ Institute of Pathology Klinikum Leverkusen, Am Gesundheitspark 11, 51375 Leverkusen, Germany

${ }^{*}$ Contributed equally

Key words: HCMEC, HCTEC, hypoxia, sVEGFR-1, sVEGFR-2 human colorectal cancer and non-neoplastic colon will be useful for understanding the progressive behavior of colorectal cancer. The different secretion profiles of sVEGFR-1 and -2 between HCTEC and HCMEC underline the importance of using a functionally adequate and relevant tumor-derived microvasculature for in vitro studies of tumor progression. Since sVEGFR-1 can act as a natural endogenous VEGF-inhibitor, the homogeneously low sVEGFR-1 levels under normoxia and hypoxia in HCTEC could be a marker for a 'pro-angiogenetic disposition' of the tumorderived endothelium. The reduced sVEGFR-2 level profiles in hypoxic HCMEC and HCTEC provide evidence for a novel microvascular endothelium-specific biomarker in hypoxia-response processes.

\section{Introduction}

Tumor angiogenesis, one of the hallmarks of the cancer phenotype, is the proliferation of a network of blood vessels from a pre-existing mature vasculature that penetrates into malignant tumors or from recruited precursor endothelial cells $(1,2)$. Enhanced vascularity results in increase of tumor growth by supplying nutrients and oxygen and enhanced metastatic potential by providing the matrix for tumor cell intravasation. It is well documented that tumor vessels differ morphologically and functionally from their normal counterparts (3). Thus, tumor vessels proliferate more rapidly, are disorganized and have structural abnormalities of their mural components, irregular blood flow and elevated permeability. Additionally, tumor-derived endothelial cells overexpress specific genes, are sensitive to growth factors and resistant to apoptotic stimuli and demonstrate cytogenetic abnormalities. These significant differences between normal and tumor endothelial cells necessitate the use of endothelial cells isolated from tumor tissue for studying the mechanisms of tumor angiogenesis and endothelium-tumor interaction. Until now limited studies were carried out using cultured tumorderived endothelial cells from carcinomas (4-6).

The tumor microenvironment is characterized by hypoxia (7). The discrepancy between rapid tumor proliferation and insufficient blood supply leads to low oxygen partial pressure $\left(\mathrm{pO}_{2}\right)$ in tumors. In such an unfavourable mileau tumors can remain viable by stimulating angiogenesis predominantly by 
vascular endothelial growth factor (VEGF) $(8,9)$. VEGF binds to cognate receptor tyrosine kinases, VEGFR-1 and VEGFR-2, located on the plasma membrane of endothelial cells. VEGFR-2 functions as the major mediator of endothelial mitogenesis, survival, vascular permeability as well as migration and angiogenesis. VEGFR-1 has a dual function in angiogenesis acting in a positive or negative manner under different biological conditions (10-12). The vegfr-l gene encodes not only the mRNA for the full-length receptor but also a short mRNA for a soluble form of the VEGFR-1 (sVEGFR-1), which carries only the extracellular domain. sVEGFR-1 is secreted by endothelial cells and can act as a natural endogenous inhibitor by sequestering VEGF and by binding and inactivating membrane-bound VEGFR-1 and -2 receptors (13). The role of sVEGFR-1 as a surrogate marker for disease progression in carcinomas of different histogenetic origin is well documented (14-17). sVEGFR-1 is also a potent endogenous inhibitor of VEGF-mediated angiogenesis. Enhanced expression of sVEGFR-1 by tumor cells in vivo inhibits solid tumor growth, impedes metastatic nodule development and prolongs survival (18). Ebos et al have found that sVEGFR-2, the truncated form of the fulllength receptor VEGFR-2 is similar to sVEGFR-1, naturally occurring in mouse and human plasma, probably secreted by endothelial cells (19). Until now it is not clear whether sVEGFR-2 is a product of alternative mRNA splicing, proteolytic cleavage of the membrane receptor or another mechanism. It appears likely that sVEGFR-2 is a product of ectodomain cleavage from the cell surface. Using xenograft tumors it was shown that increasing tumor burden leads to decreased sVEGFR-2 levels mediated by tumor-derived VEGF (20). Experiments with recombinant sVEGFR-2 capable of neutralizing VEGF action in vitro could also block tumor angiogenesis in vivo (21). Currently, data on secretion of sVEGFR-1 and -2 from isolated tumor-derived endothelial cells has not been described.

Previously, we established a method for isolation of human colonic microvascular endothelial cells (HCMEC) (22). The main goal of the present study was to establish a relatively easy, reproducible method for isolation of human colonic tumor-derived microvascular endothelial cells (HCTEC) for comparative studies. In a next step we compared HCMEC and HCTEC, each obtained from the same patient, with respect to their ability to secrete sVEGFR-1 and -2 under normoxic and hypoxic culture conditions.

\section{Materials and methods}

Isolation and culture of HCTEC. Human colonic tumorderived endothelial cells were obtained from central nonnecrotic tumor areas of colon carcinomas surgically resected from patients who underwent colectomies for colon cancer. The present study was approved by the ethics committee of the University of Mainz and informed consent as defined by the Helsinki Declaration was obtained from each patient. Tumor tissue $(\sim 3 \mathrm{~g})$ was cut into small fragments with scissors. After removal of blood cells with PBS-BSA $0.1 \%$, the tissue was treated with dispase $\left(0.4 \%\right.$ at $4^{\circ} \mathrm{C}$ for $18 \mathrm{~h}$; Sigma, Taufkirchen, Germany). After filtration the tissue was digested with collagenase type II $\left(0.2 \%\right.$, at $37^{\circ} \mathrm{C}$ for $40 \mathrm{~min}$;
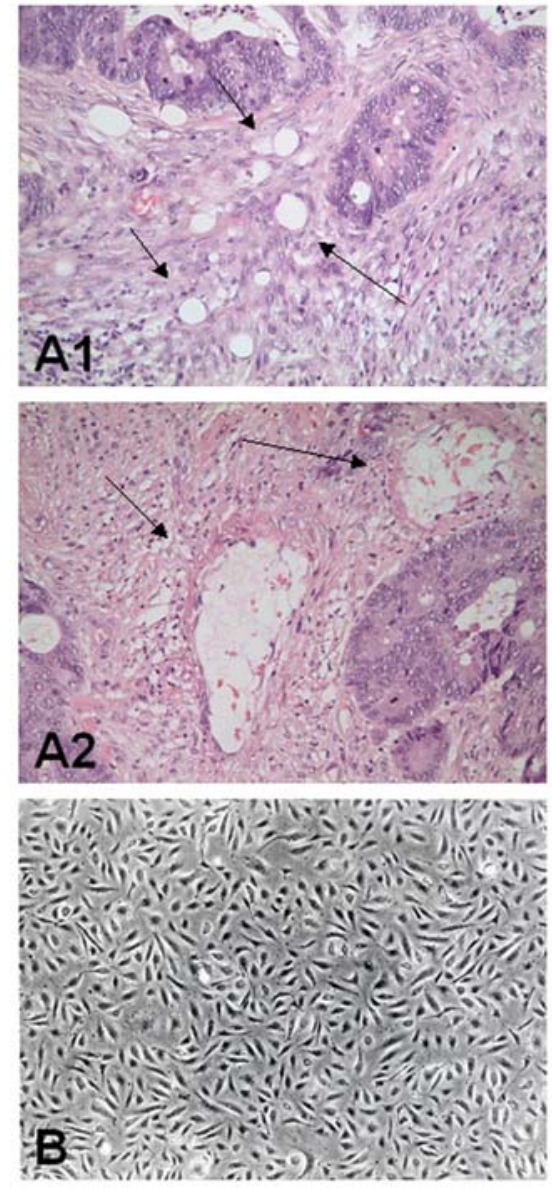

Figure 1. Morphology of colonic tumor-derived microvasculature in situ and in vitro. (A) Light microscopy of central, non-necrotic tumor areas with abundant lymphatic (A1) and blood (A2) microvessels (arrows), from which HCTEC cultures are obtained (magnification, x400). (B) Phase contrast microscopy of cultured HCTEC as confluent monolayer of closely associated fusiform-shaped endothelial cells with rosette-like arrangement.

Serva, Heidelberg, Germany) in a volume of $10 \mathrm{ml}$, followed by a further filtration step. The cell clumps were then repeatedly resuspended in PBS-BSA and filtrered through a $100 \mu \mathrm{m}$ cell strainer. The cell suspension was then centrifuged for $5 \mathrm{~min}$ at $1500 \mathrm{rpm}$ and the cell pellet was resuspended in culture medium composed of MCDB131 with $15 \%$ fetal calf serum (FCS; Gibco, BRL, UK), Glutamax $1 \%$ (Gibco), Fungizone 1\% (Gibco) and Ciprobay 1\% (Bayer, Leverkusen, Germany) and seeded in gelatin-coated $(0.2 \%)$ tissue culture flasks. This mixed cell culture was cultivated at $37^{\circ} \mathrm{C}$ in a gas mixture of $5 \% \mathrm{CO}_{2}$ in air for $4 \mathrm{~h}$, followed by washing with PBS to remove all non-adherent cells. Fresh medium (Endothelial Growth Medium and supplement mix; Promocell, Heidelberg, Germany) was added and cells were cultivated as described above until subconfluence. The monolayer was subcultured 1:3 by trypsinization. Positive selection of HCTEC was achieved using magnetic beads ( $1 \mu \mathrm{m}$ diameter) coated with a mouse monoclonal antibody against CD31 (Dynal, Oslo, Norway) in passage one and two. In the third passage culture medium was changed to MCDB131 with $15 \%$ FCS, $1 \%$ glutamax, $1 \%$ penicillin/streptomycin, $2 \mathrm{ng} / \mathrm{ml}$ bFGF (Sigma), $50 \mu \mathrm{g} / \mathrm{ml}$ heparin (Sigma), and $50 \mu \mathrm{g}$ ECGS (Sigma). 
A
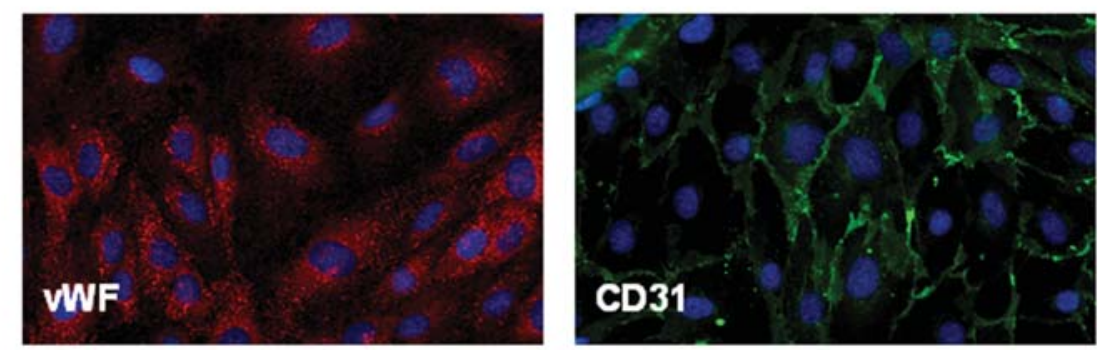

B
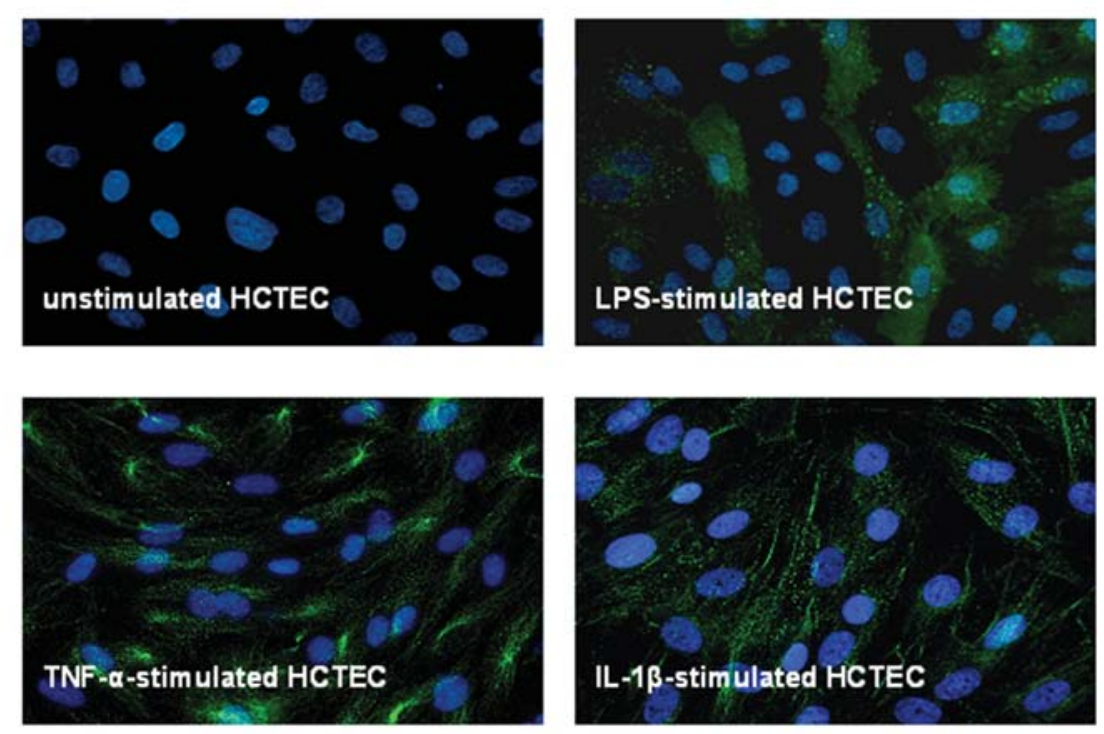

Figure 2. Characterization of HCTEC by using immuno-fluorescence microscopy. (A) Detection of von Willebrand factor (vWF) showed specific cytoplasmatic, granular staining pattern and CD31 showed specific surface expression. (B) Cytokine- and LPS-inducible expression of E-selectin in HCTEC: Whereas non-stimulated HCTEC do not express E-selectin, an inducible expression was found after stimulation of HCTEC with LPS, TNF- $\alpha$ and IL-1ß.

Characterization of HCTEC. For immunofluorescence staining HCTEC were seeded on fibronectin-coated glass chamber slides (LabTec, Nunc, Wiesbaden, Germany) and grown to subconfluence. Cells were fixed with buffered $3.7 \%$ paraformaldehyde (15 min, room temperature) and permeabilized with $0.1 \%$ Triton $\mathrm{X}-100$ (5 min, room temperature). Monoclonal mouse anti-human E-Selectin (Serva), monoclonal mouse anti-human CD31 (Dako, Hamburg, Germany), polyclonal rabbit anti-human Faktor VIII (Dako) and monoclonal mouse anti-human sm-actin (Progen, Heidelberg, Germany), monoclonal mouse antihuman cytokeratin (Dako) were used as primary antibodies. Alexa Fluor 488 goat anti-mouse and Alexa 546 goat antirabbit (Eugene, OR, USA) were used as secondary antibodies. Nuclear staining was performed with Hoechst 33342 (Sigma). Fluorescently labeled cells were covered with GelMount (Biomeda Corp/Natutec, Frankfurt, Germany).

For the cytokine and LPS stimulation experiments of Eselectin in HCTEC, endothelial cells were stimulated with $50 \mathrm{ng} / \mathrm{ml}$ LPS (Sigma), $20 \mathrm{ng} / \mathrm{ml} \mathrm{TNF-} \alpha$ (Sigma) and $0.5 \mathrm{ng} / \mathrm{ml}$ IL-1ß (Strathmann Biotech, Hamburg, Germany) for $6 \mathrm{~h}$.
Isolation and culture of HCMEC. HCMEC were isolated from the corresponding normal colonic tissue of the same donor and cultured as previously described (22). For all experiments HCMEC in passage 3 were used.

Immunofluorescence. HCTEC and HCMEC were seeded onto fibronectin-coated glass chamber-slides (LabTek). After growing to subconfluence cells were fixed with buffered $3.7 \%$ paraformaldehyde (15 min, room temperature) and permeabilized with $0.1 \%$ Triton X-100 (5 min, room temperature). Rabbit polyclonal anti-human FLT-1 (Santa Cruz, Heidelberg, Germany) and monoclonal anti-human KDR (Chemicon, Düsseldorf, Germany) were used as primary antibodies. Alexa Fluor 488 goat anti-mouse and Alexa 546 goat anti-rabbit were used as secondary antibodies. Nuclear staining was performed with Hoechst 33342 (Sigma). Fluorescently labeled cells were covered with GelMount.

Semiquantitative reverse transcription-PCR. RNA isolation was performed using the RNeasy kit (Qiagen, Hilden, Germany) in accordance with the manufacturer's protocols. 
B-actin and VEGFR-1 and -2 transcripts were analyzed by RT-PCR. RT was performed with the help of the Omniscript RT kit (Qiagen) in accordance with the manufacturer's manual. As a template for the specific PCR reactions $2 \mu 1$ of the synthesized cDNAs-pool was used. To amplify the B-actin specific fragment, 574 base pair (bp), the primers 5'-GACCTGACTGACTACCTCATGA-3' (forward) and 5'-AGCATTTGCGGTGGACGATGGAG-3' (reverse) were used. Amplification of the human VEGFR-1 specific fragment, $441 \mathrm{bp}$, was performed using the primers 5'-GCACCT TGGTTGTGGCTGA-3' (forward) and 5'-GGTTTCGCA GGAGGTATGGTG-3' (reverse). Amplification of the human VEGFR-2 specific fragment, $473 \mathrm{bp}$, was performed using the primers 5'-TATGTCTATGTTCAAGATTAC-3' (forward) and 5'-AAGTTTCTTATGCTGATGCTT-3' (reverse). Amplification reactions were performed with the help of the Gene Amplification PCR System 2400 (Perkin Elmer, Norwalk, CT, USA) thermocycler. The PCR products were separated on agarose gels $(2 \%)$ supplemented with ethidium bromide and analyzed by viewing under UV.

ELISA analysis. Levels of sVEGFR-1 and sVEGFR-2 were measured in cell lysates of HCMEC and HCTEC of 5 patients cultivated under normoxic $\left(21 \% \mathrm{O}_{2}\right)$ and hypoxic $\left(3 \% \mathrm{O}_{2}\right)$ conditions over $24 \mathrm{~h}$. For the quantitative determination of human sVEGFR-1 and -2 concentrations in cell lysates commercially available sandwich ELISA assays (R\&D Systems, Wiesbaden, Germany) were used according to the manufacturer's instructions. Cell lysates were prepared using a lysis buffer provided with the kit. The optic density of the color reaction was determined using a microplate ELISA reader set to $450 \mathrm{~nm}$.

Statistical analysis. Statistical analyses were performed using the Wilcoxon signed rank test. Significance was set at $\mathrm{p}<0.05$.

\section{Results}

Isolation and characterization of human colonic tumorderived endothelial cells (HCTEC). HCTEC are isolated from the carcinoma tissue from specimens of five patients undergoing surgery for colorectal cancer. HCTEC were obtained from lymphatic and blood microvessels from central non-necrotic tumor areas (Figs. 1A1 and 2). The isolation was carried out after dispase/collagenase digestion and incubation with CD31-coated magnetic beads. The isolated endothelial cells were cultured in gelatin-coated flasks and grown to confluence within 6-8 days. The culture monolayer of the HCTEC had the same morphology as the normal counterpart HCMEC and consisted of closely associated cells with a fusiform shape (Fig. 1B). The cultured cells were assayed for an endothelial-specific phenotype in the third passage of cultivation, leaving enough numbers of cells for further experiments. To define the endothelial origin of HCTEC, expression analyses were performed using immunofluorescence staining. Most of the endothelial cells specifically expressed the endothelial markers, von Willebrand factor and CD31 (Fig. 2A), whereas the reaction with antibodies to whole-cytokeratin and sm-actin were
A VEGFR-1 VEGFR-2

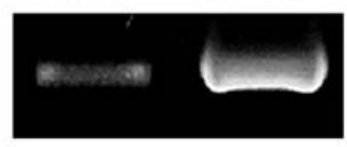

$\beta$-actin

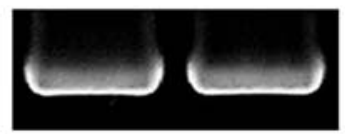

B

VEGFR-1

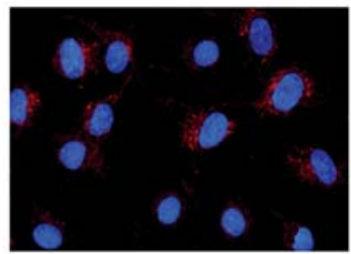

VEGFR-2

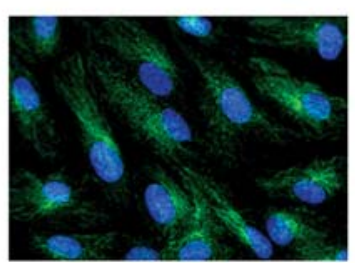

Figure 3. RT-PCR and immunofluorescence for VEGFR-1 and -2 expression in HCTEC. (A) VEGFR-1 and -2 m-RNA is detected in HCTEC. The quality of the reaction was judged upon amplification of a specific B-actin fragment. (B) VEGFR-1 and -2 proteins are also detected in HCTEC.

negative, a result that excludes the possibility of contamination with epthelial cells and subendothelial smooth muscle cells (data not shown). Culture impurity with fibroblasts can also be excluded because of rapid overgrowth of this cell type. In a next step of characterization, we investigated the induction of E-selectin, a member of the selectin family of endothelial cell adhesion molecules (23), on HCTEC. E-selectin is a key endothelial marker because it is generally expressed only in endothelial cells in response to induction by pro-inflammatory factors, such as tumor necrosis factor- $\alpha$ (TNF- $\alpha)$, interleukin 1- $\beta$ (IL-1ß) and bacterial lipopolysaccharide (LPS) $(24,25)$. Fig. 2B demonstrates the inducibility of E-selectin on HCTEC after stimulation with TNF- $\alpha$, IL-1ß and LPS. Expression of Eselectin was not detected in non-stimulated HCTEC.

Using RT-PCR and immunfluorencense constitutive expression of VEGFR-1 and VEGFR-2 mRNA and protein was found. The mRNA levels of VEGFR-1 were lower in comparison with VEGFR-2 (Fig. 3A). At the protein level congruent results were obtained (Fig. 3B).

For the following comparative studies, using the same protocol we isolated from the non-neoplastic colon tissue pairwise the corresponding HCMEC from the normal microvasculature.

sVEGFR-1 and -2 are differentially expressed in HCMEC and HCTEC. In the next step, we compared the concentrations of sVEGFR-1 and sVEGFR-2 in cell lysates 

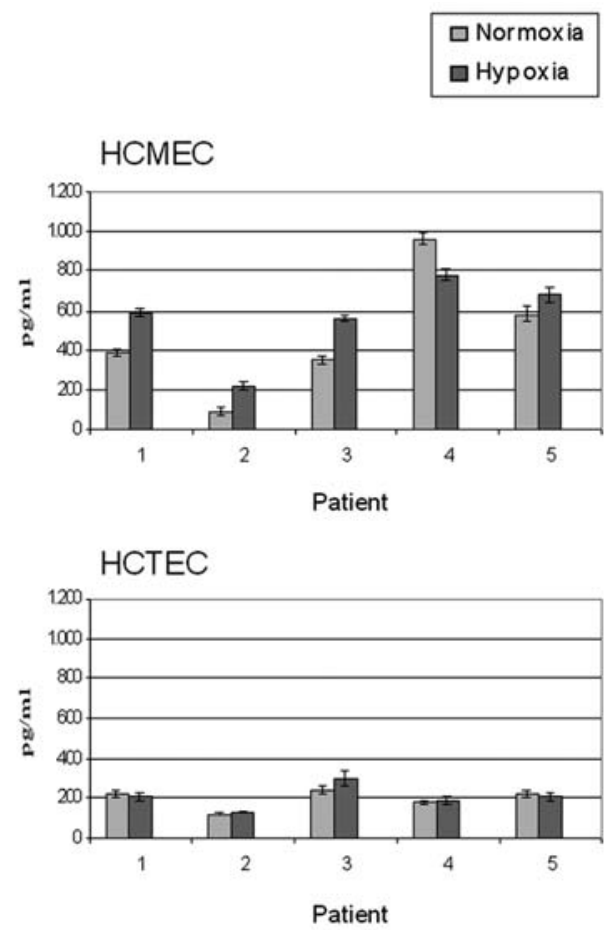

Figure 4. Determination of sVEGFR-1 concentrations by ELISA in HCMEC and corresponding HCTEC from five patients. Comparison of normoxic $\left(21 \% \mathrm{O}_{2}\right)$ and hypoxic $\left(3 \% \mathrm{O}_{2}\right)$ conditions $(\mathrm{n}=3$, mean values \pm standard error).

of HCMEC and HCTEC of five patients under normoxia and hypoxia using specific ELISA. Under hypoxic conditions a marked increase of sVEGFR-1 on HCMEC in three of the five investigated cases was observed (range: $90-390 \mathrm{pg} / \mathrm{ml}$ protein under normoxia vs. $220-590 \mathrm{pg} / \mathrm{ml}$ under hypoxia) (Fig. 4). Additionally, whereas in one case the sVEGFR-1 levels remained unchanged under normoxia and hypoxia $(580 \mathrm{pg} / \mathrm{ml}$ protein under normoxia vs. $610 \mathrm{pg} / \mathrm{ml}$ under hypoxia), in one individual hypoxia induced sVEGFR-1 reduction (960 pg/ml protein under normoxia vs. $780 \mathrm{pg} / \mathrm{ml}$ protein under hypoxia). In contrast, in HCTEC the sVEGFR-1 expression pattern remained unchanged unter hypoxia (range: $120-240 \mathrm{pg} / \mathrm{ml}$ protein under normoxia vs. $130-300 \mathrm{pg} / \mathrm{ml}$ protein under hypoxia). Very similar mean values for sVEGFR-1 levels were found under both oxygen conditions (196 pg/ml protein under normoxia versus $208 \mathrm{pg} / \mathrm{ml}$ under hypoxia). In comparison to HCMEC markedly lower sVEGFR-1 protein concentrations were found in the tumor-derived endothelium under both oxygen conditions. Thus, mean values for sVEGFR-1 levels in HCMEC were $474 \mathrm{pg} / \mathrm{mg}$ protein under normoxia and $566 \mathrm{pg} / \mathrm{mg}$ under hypoxia and in HCTEC $196 \mathrm{pg} / \mathrm{mg}$ protein under normoxia and $208 \mathrm{pg} / \mathrm{mg}$ under hypoxia.

In three of five investigated cases sVEGFR-2 was significantly reduced in HCMEC under hypoxia $(\mathrm{p}=0.001)$ (Fig. 5). The means of sVEGFR-2 levels were $2000 \mathrm{pg} / \mathrm{mg}$ protein under normoxia and $1620 \mathrm{pg} / \mathrm{mg}$ under hypoxia. Four hypoxic HCTEC cultures expressed reduced levels of sVEGFR-2 (mean levels $2540 \mathrm{pg} / \mathrm{ml}$ protein under normoxia vs. $1660 \mathrm{pg} / \mathrm{ml}$ protein under hypoxia; $\mathrm{p}<0.001)$.
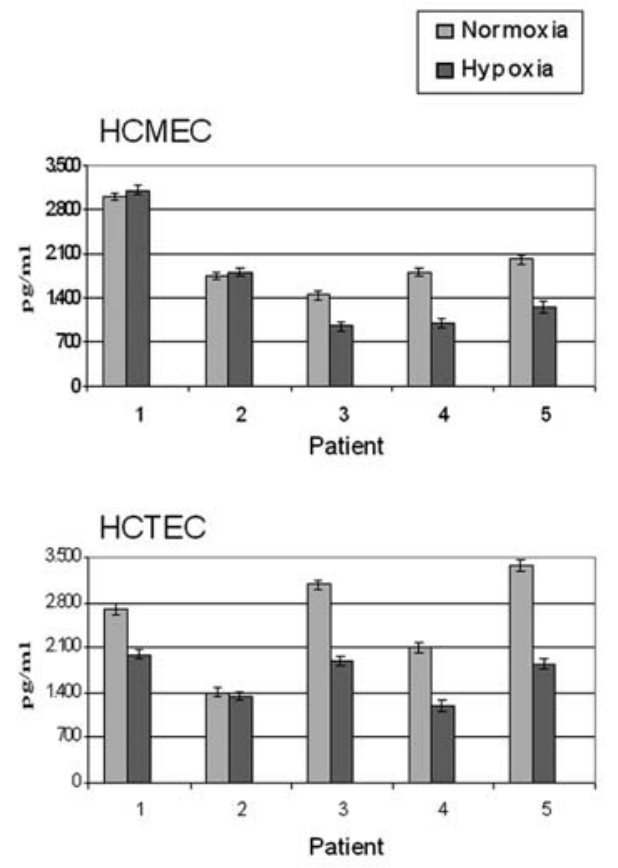

Figure 5. Determination of sVEGFR-2 concentrations by ELISA in HCMEC and corresponding HCTEC from five patients. Comparison of normoxic $\left(21 \% \mathrm{O}_{2}\right)$ and hypoxic $\left(3 \% \mathrm{O}_{2}\right)$ conditions $(\mathrm{n}=3$, mean values \pm standard error).

sVEGFR-2 was significantly elevated in two cases (patients 3 and 5) and reduced in one case (patient 2) in HCTEC in comparison to the corresponding HCMEC under both conditions of normoxia and hypoxia. Following exposure of HCTEC to hypoxia a marked reduction of sVEGFR-2 in one case was observed, whereas protein levels remained unchanged under normoxia (patient 1). HCMEC and HCTEC of patient 4 have exhibited the same sVEGFR-2 concentrations under both oxygen conditions. Thus, the normoxic and hypoxic sVEGFR-2 expression pattern of HCTEC and corresponding HCMEC was variable and individually different. Interestingly, the sVEGFR-2 level changes of HCTEC and the corresponding HCMEC of the same patient were unidirectional under normoxia and hypoxia in four cases (patient 2,3,4 and 5).

\section{Discussion}

Endothelial cells in the vicinity of tumors make an essential contribution to tumor growth and metastasis. It is now well recognized that tumor endothelium is different from normal vasculature with respect to morphology and biological behavior. The elucidation of the mechanisms of tumor angiogenesis will certainly provide more precise and effective anticancer therapies. To this end comparative studies of the characteristic vasculature from the tumor and the corresponding non-neoplastic tissue are imperative. Previously, we described a method for the isolation and culture of human colonic microvascular endothelial cells (HCMEC). In the present study we extended this approach to the isolation of tumor-derived endothelial cells from specimens of surgically resected colon carcinoma (HCTEC). By using CD31-coated 
magnetic beads we selectively isolated microvascular endothelial cells from central non-necrotic tumor areas. The method is reproducible, relatively easily applicable and yielded endothelial cells with a high viability. Morphologically the endothelial cell colonies in culture exhibited a fusiform appearance, which was also shown in HCMEC. The endothelial nature of the cells was demonstrated phenotypically by positive immunostaining with endothelial specific antibodies to vWF and CD31.

There is evidence that E-selectin is involved in the process of colon carcinoma metastasis by mediating adhesion between the endothelium and the corresponding ligand on colon carcinoma cells (26). In the present study E-selectin was expressed on HCTEC after stimulation with LPS, TNF- $\alpha$ and IL-1ß. These results are in agreement with the observation of Schellerer et al, who found E-selectin induction in IL-1ß-stimulated tumor-derived endothelial cells from colon carcinomas (6). In both culture studies the basal E-selectin level under unstimulated conditions was negative. However, Ye et al reported that E-selectin was constitutively expressed in vessels of colorectal cancer specimens (27). These observations indicate that for the in vivo situation the totality of components of the tumor microenvironment should be taken into consideration. Thus, colon carcinomas are characterized by peritumoral inflammation with secretion of high amounts of pro-inflammatory cytokines, such as TNF- $\alpha$ IL-1ß. LPS, an endotoxin of ubiquitously existing colonic bacteria, which can translocate from the luminal site to the site of deepest tumor penetration after intestinal mucosal injury such as tumor ulceration (28). Taking these facts together, our present results indicate that a prolonged peritumoral availability of LPS, TNF- $\alpha$ and IL- $1 \beta$ could potentially stimulate E-selectin expression in tumor endothelium and subsequently an E-selectin-mediated metastasis cascade.

We observed that HCTEC constitutively coexpressed mRNA and protein of both VEGF receptors, VEGFR-1 and -2. In particular, VEGFR-1 was expressed in markedly lower levels in comparison with VEGFR-2. This observation is consistent with previously published data in tumor-derived endothelial cells isolated from human renal carcinomas (4). The survival and growth of tumor cells is dependent on adequate supplies of oxygen and nutrients controlled by tumor angiogenesis. The extent of angiogenesis results from the balance between pro-angiogenetic and anti-angiogenetic factors released by tumor and host cells (29). VEGFR-2 is the major mediator of endothelial mitogenesis and survival as well as angiogenesis. In contrast, VEGFR-1 functions mostly as a negative regulator of angiogenesis. In this context, the ratio of high VEGFR-2 levels to low VEGFR-1 levels in the basal status of HCTEC may reflect the expected proangiogenic phenotype of the tumor vasculature.

Studies concerning the secretion of sVEGFR-1 and - 2 from tumor-derived endothelial cells have not yet been published. The results presented here clearly show that both soluble VEGF receptors are detectable in HCTEC cultures. However, in comparison with HCMEC markedly lower sVEGFR-1 protein concentrations were found in HCTEC. These low sVEGFR-1 levels remain unchanged in HCTEC under hypoxia. Since sVEGFR-1 is a potent and selective endogenous inhibitor of VEGF-mediated angiogenesis, this finding suggests that the pro-angiogenic status of the tumor endothelium in normoxic and hypoxic tumor areas can also be sustained by low sVEGFR-1 levels. Additionally, the unchanged sVEGFR-1 expression pattern of HCTEC under normoxia and hypoxia could be a marker of their 'hypoxia-resistance'.

In contast to sVEGFR-1, the (patho)physiological roles of sVEGFR-2 still remain unknown. Our previous study provided evidence that hypoxia consistently induces a decrease of sVEGFR-2 levels in HCMEC (22). In accordance, in the present study hypoxic HCMEC cultures also demonstrated a significant sVEGFR-2 reduction. Interestingly, sVEGFR-2 decrease in response to hypoxia was also found in the corresponding tumor endothelium. Our hypothesis, that sVEGFR-2 can serve as a microvascular endothelium-specific biomarker in hypoxia-response processes seems to be valid for tumor microvasculature as well. The generally accepted opinion is that an increase in circulating VEGF leads to a corresponding decrease in sVEGFR-2 level by internalization and down-regulation of the receptor from the cell surface (20). Since hypoxia is the major inducer of the highly potent pro-angiogenic and prosurvival endothelial mediator VEGF, hypoxia-induced reduction of sVEGFR-2 in HCTEC may be an accompanying pathophysiological event of VEGF-mediated processes of tumor progression. Interestingly, the secretion profiles of sVEGFR-2 between HCTEC and HCMEC under normoxia and hypoxia were variable. Whether this observation is dependent on different aggressive and/or metastatic tumor potential needs to be confirmed by further studies.

We conclude that comparative studies with endothelial cells of human colorectal cancer and healthy colon will be useful for understanding the progressive behavior of colorectal cancer. The homogeneously low sVEGFR-1 levels under normoxia and hypoxia in HCTEC could be a marker for a 'pro-angiogenetic disposition' of the tumorderived endothelium. Similar to HCMEC, sVEGFR-2 can serve as a microvascular endothelium-specific biomarker in hypoxia-response processes in HCTEC. On the basis of our studies we suggest that hypoxia-induced reduction of sVEGFR-2 could facilitate the VEGF-mediated processes of tumor progression.

\section{Acknowledgements}

This study was funded by the state of Rhineland-Palatine, Germany within the scope of the general funds of the Institute of Pathology at the Johannes Gutenberg University in Mainz for personnel and consummable costs.

\section{References}

1. Hanahan D and Folkman J: Patterns and emerging mechanisms of the angiogenic switch during tumorigenesis. Cell 86: 353-364, 1996.

2. Ribatti D: The involvement of endothelial progenitor cells in tumor angiogenesis. J Cell Mol Med 8: 294-300, 2004.

3. Hida K, Hida Y and Shindoh M: Understanding tumor endothelial cell abnormalities to develop ideal anti-angiogenic therapies. Cancer Sci 99: 459-466, 2007.

4. Bussolati B, Deambrosis I, Russo S, Deregibus MC and Camussi G: Altered angiogenesis and survival in human tumorderived endothelial cells. FASEB J 17: 1159-1161, 2003. 
5. Allport JR and Weissleder R: Murine Lewis lung carcinomaderived endothelium expresses markers of endothelial activation and requires tumor-specific extracellular matrix in vitro. Neoplasia 5: 205-217, 2003.

6. Schellerer VS, Croner RS, Weinländer K, Hohenberger W, Stürzl M and Naschberger E: Endothelial cells of human colorectal cancer and healthy colon reveal phenotypic differences in culture. Lab Invest 87: 1159-1170, 2007.

7. Toffoli S and Michiels C: Intermittent hypoxia is a key regulator of cancer cell and endothelial cell interplay in tumours. FEBS J 275: 2991-3002, 2008.

8. Ferrara N: Vascular endothelial growth factor: basic science and clinical progress. Endocr Rev 25: 581-611, 2004.

9. Ferrara N, Gerber HP and LeCouter J: The biology of VEGF and its receptors. Nat Med 9: 669-676, 2003.

10. Shibuya M: Differential roles of vascular endothelial growth factor receptor- 1 and receptor-2 in angiogenesis. J Biochem Mol Biol 39: 469-478, 2006.

11. Hiratsuka S, Nakamura K, Iwai S, Murakami M, Itoh T, Kijima H, Shipley JM, Senior RM and Shibuya M: MMP9 induction by vascular endothelial growth factor receptor- 1 is involved inlung-specific metastasis. Cancer Cell 2: 289-300, 2002.

12. Carmeliet P, Moons L, Luttun A, Vincenti V, Compernolle V, De Mol M, Wu Y, Bono F, Devy L, Beck H, Scholz D, Acker T, DiPalma T, Dewerchin M, Noel A, Stalmans I, Barra A, Blacher S, Vandendriessche T, Ponten A, Eriksson U, Plate KH, Foidart JM, Schaper W, Charnock-Jones DS, Hicklin DJ, Herbert JM, Collen D and Persico MG: Synergism between vascular endothelial growth factor and placental growth factor contributes to angiogenesis and plasma extravasation in pathological conditions. Nat Med 7: 575-583, 2001.

13. Shibuya M: Structure and dual function of vascular endothelia growth factor receptor-1 (Flt-1). Int J Biochem Cell Biol 33: 409-420, 2001.

14. Artini PG, Ruggiero M, Monteleone P, Carpi A, Cristello F, Cela V and Genazzani AR: Vascular endothelial growth factor and its soluble receptor in benign and malignant ovarian tumors. Biomed Pharmacother 62: 373-377, 2007.

15. Yilmaztepe A, Ulukaya E, Zik B, Yagci A, Sevimli A, Yilmaz M, Erdogan BB, Koc M, Akgoz S, Karadag M and Tokullugil A: Soluble vascular endothelial growth factor receptor-1 (sVEGFR-1) is decreased in lung cancer patients showing progression: a pilot study. Cancer Invest 25: 322-327, 2007.

16. Bando H, Weich HA, Brokelmann M, Horiguchi S, Funata N, Ogawa T and Toi M: Association between intratumoral free and total VEGF, soluble VEGFR-1, VEGFR-2 and prognosis in breast cancer. Br J Cancer 92: 553-561, 2005.

17. Chin KF, Greenman J, Reusch P, Gardiner E, Marme D and Monson JR: Vascular endothelial growth factor and soluble Tie-2 receptor in colorectal cancer: associations with disease recurrence. Eur J Surg Oncol 29: 497-505, 2003.
18. Goldman CK, Kendall RL, Cabrera G, Soroceanu L, Heike Y, Gillespie GY, Siegal GP, Mao X, Bett AJ, Huckle WR, Thomas KA and Curiel DT: Paracrine expression of a native soluble vascular endothelial growth factor receptor inhibits tumor growth, metastasis, and mortality rate. Proc Natl Acad Sci USA 95: 8795-8000, 1998.

19. Ebos JM, Bocci G, Man S, Thorpe PE, Hicklin DJ, Zhou D Jia $\mathrm{X}$ and Kerbel RS: A naturally occurring soluble form of vascular endothelial growth factor receptor 2 detected in mouse and human plasma. Mol Cancer Res 2: 315-326, 2004.

20. Ebos JM, Lee CR, Bogdanovic E, Alami J, Van Slyke P, Francia G, Xu P, Mutsaers AJ, Dumont DJ and Kerbel RS: Vascular endothelial growth factor-mediated decrease in plasma soluble vascular endothelial growth factor receptor-2 levels as a surrogate biomarker for tumor growth. Cancer Res 68: 521-529, 2008.

21. Lin P, Sankar S, Shan S, Dewhirst MW, Polverini PJ, Quinn TQ and Peters KG: Inhibition of tumor growth by targeting tumor endothelium using a soluble vascular endothelial growth factor receptor. Cell Growth Differ 9: 49-58, 1998.

22. Jayasinghe C, Simiantonaki N, Michel-Schmidt R and Kirkpatrick CJ: Hypoxia-induced reduction of sVEGFR-2 levels in human colonic microvascular endothelial cells (HCMEC) in vitro: Comparative study with HUVEC. Int J Mol Med 23: 49-55, 2009.

23. Tedder TF, Steeber DA, Chen A and Engel P: The selectins: vascular adhesion molecules. FASEB J 9: 866-873, 1995.

24. Wong D and Dorovini-Zis K: Regualtion by cytokines and lipopolysaccharide of E-selectin expression by human brain microvessel endothelial cells in primary culture. J Neuropathol Exp Neurol 55: 225-235, 1996.

25. Haraldsen G, Kvale D, Lien B, Farstad IN and Brandtzaeg P: Cytokine-regulated expression of E-selectin, intercellular adhesion molecule-1 (ICAM-1), and vascular cell adhesion molecule-1 (VCAM-1) in human microvascular endothelial cells. J Immunol 156: 2558-2565, 1996.

26. Ugorski M and Laskowska A: Sialyl Lewis(a): a tumorassociated carbohydrate antigen involved in adhesion and metastatic potential of cancer cells. Acta Biochim Pol 49: 303-311, 2002.

27. Ye C, Kiriyama K, Mistuoka C, Kannagi R, Ito K, Watanabe T, Kondo K, Akiyama S and Takagi H: Expression of E-selectin on endothelial cells of small veins in human colorectal cancer. Int J Cancer 61: 455-560, 1995.

28. Schoeffel U, Pelz K, Häring RU, Amberg R, Schandl R, Urbaschek R, von Specht BU and Farthmann EH: Inflammatory consequences of the translocation of bacteria and endotoxin to mesenteric lymph nodes. Am J Surg 180: 65-72, 2000.

29. Ellis LM, Liu W, Ahmad SA, Fan F, Jung YD, Shaheen RM and Reinmuth $\mathrm{N}$ : Overview of angiogenesis: Biologic implications for antiangiogenic therapy. Semin Oncol 28: 94-104, 2001. 